\title{
Erratum
}

\section{QCD and QED corrections to the longitudinal polarization asymmetry}

S. Jadach, J.H. Kühn, R.G. Stuart, Z. Was

Z. Phys. C - Particles and Fields 38, 609 (1988)

Equation (22) should be replaced by

$\left.\sigma_{R R}=\sigma_{L L}=\frac{2 \alpha}{\pi} \int_{k_{\min }}^{k_{\max }} d k k \sigma[s 1-k)\right]$

where $\sigma$ denotes the cross section for unpolarized beams.
The subsequent discussion and Fig. 8 remain unchanged.

J.K. thanks M. Einhorn for drawing his attention to this point and A. Denner for discussions. 